\title{
Polineuropatia no Paciente Crítico: Um Diagnóstico Comum em Medicina Intensiva?*
}

\author{
Polyneuropathy in the Critical III patient: \\ A common Diagnosis in Intensive Care Medicine?
}

\author{
Rafael Fernando Brandão Canineu', Marcella M. Cabral2, Hélio Penna Guimarães ${ }^{3,4}$, \\ Renato Delascio Lopes ${ }^{3}$, Letícia Sandre Vendrame Saes ${ }^{3,4}$, Antonio Carlos Lopes ${ }^{4,5}$
}

\section{RESUMO}

JUSTIFICATIVA E OBJETIVOS: A polineuropatia axonal difusa, hoje mais conhecida como polineuropatia do paciente crítico (PPC), tem sido relatada por autores há décadas, porém, apenas nos últimos 30 anos, ocupa maior importância como causa de dependência prolongada de ventilação mecânica, em pacientes gravemente enfermos internados em Unidades de Terapia Intensiva. Esta revisão teve por objetivo apresentar os princípios tópicos que norteiam a fisiopatologia, diagnóstico e tratamento desta doença em Medicina intensiva.

CONTEÚDO: A importância da PPC como complicação inicial do choque séptico e em pacientes com disfunção de múltiplos de órgãos e sistemas (DMOS) está claramente descrita como responsável pelo prolongamento da permanência na UTI e, também pela redução gradativa da probabilidade de sobrevida. Su-

1. Médico Especializando da Disciplina de Clinica Médica da UNIFESP-EPM

2. Fisioterapeuta da UTI da Disciplina de Clinica Médica da UNIFESP-EPM

3. Médico Assistente da UTI da Disciplina de Clinica Médica da UNIFESP-EPM

4. Titulo de Especialista em Medicina Intensiva pela AMIB-AMB

5. Professor Titular de Disciplina de Clinica Médica da UNIFESPEPM

${ }^{*}$ Recebido da UTI da Disciplina de Clinica Médica da Universidade Federal de São Paulo, Escola Paulista de Medicina (UNIFESP-EPM), São Paulo, SP

Apresentado em 20 de fevereiro de 2006

Aceito para publicação em 14 de agosto de 2006

Endereço para correspondência:

Dr. Rafael Fernando Brandão Canineu

Rua Napoleão de Barros, 715, 3A

Hospital São Paulo-UNIFESP-EPM

E-mail: rcanineu@yahoo.com.br

(C)Associação de Medicina Intensiva Brasileira, 2006 gere-se que a polineuropatia esteja relacionada com as citocinas envolvidas na sepse, além de outros mediadores que aumentariam a permeabilidade dos vasos, resultando em edema endoneural e lesão axonal. Seu início é de difícil diagnóstico, geralmente sendo possível apenas quando as complicações da sepse ou falência de múltiplos órgãos tenham sido adequadamente controladas. O diagnóstico é feito através da eletroneuromiografia. Apesar de ainda não haver nenhum tratamento medicamentoso efetivo, além do controle da doença de base, é censo comum, entre equipes multidisciplinares que o desenvolvimento da PPC não deve ser entendido como forma de reduzir os esforços do tratamento.

CONLUSÕES: A despeito de sua prevalência, ainda permanecem desconhecidos os fatores claramente associados à sua fisiopatologia, bem como adequada terapia para o manuseio desta condição.

Unitermos: Medicina Intensiva, paciente crítico, Polineuropatia

\section{SUMMARY}

BACKGROUND AND OBJECTIVES: The diffuse axonal polyneuropathy, more commonly known as Critical Illness Polyneuropathy (CIP), has been discussed by authors by decades; however, it has only been deeply studied over the last thirty years, becoming more important as an important cause of long term dependence on mechanical ventilation by seriously ill patients in intensive care medicine.

CONTENTS: A significant reason for such interest is due to the importance of the CIP as complication of the septic shock and in patients with multiple organ failure, as much as responsible for the prolonging hospitalization in the Intensive Care Unit, as for the gradual reduction of the chance of survival. It has been suggested that the polyneuropathy is related with cytokines and 
other mediators which would increase the permeability of the vases, resulting in endoneural edema and causing the axonal injury. It is difficult to do the initial diagnostic, which, in general, are only possibly recognized when the sepsis complications or the multiple organs failure have been satisfactorily controlled. The diagnosis is made through the eletroneuromiography exam, and although there is still no effective drug treatment other than the control of the basic illness, it is consensus among multidisciplinary team that the development of the CIP does not have to be understood as a way to reduce the intensity of treatment.

CONCLUSIONS: Spit of your prevalence, it is still unknown the mainly factors which are physiopathology associated as soon as your correct therapy.

Key Words: critical illness, Intensive Care, Polyneuropathy

\section{INTRODUÇÃO}

Desde os anos 1970, as desordens neuromusculares têm sido reconhecidas como a principal causa de fraqueza generalizada e dependência prolongada de ventilação mecânica, em pacientes gravemente enfermos internados em unidades de terapia intensiva (UTI). Neste grupo de pacientes, o prolongamento e a evolução da condição que motivou sua internação e a terapia instituída podem propiciar o desenvolvimento da "Polineuropatia do Paciente Crítico" (PPC), uma doença ainda não totalmente elucidada em sua fisiopatologia e etiopatogenia.

Há clara relação entre a PPC como complicação do choque séptico disfunção de múltiplos órgãos e sistemas (DMOS) ${ }^{1}$, promovendo prolongamento da permanência na UTI e redução gradativa da probabilidade de sobrevida. Nesta revisão, os artigos foram selecionados na base eletrônica MedLine, no período de 1980 à 2006, selecionando-se para apresentação apenas os artigos mais relevantes.

\section{PRIMEIROS REGISTROS E FATOS HISTÓRICOS}

O termo "Polineuropatia do Paciente Crítico" (PPC) foi inicialmente introduzido por Bolton e col. ${ }^{1}$ em 1984, quando definiram um quadro predominantemente motor, de natureza axonal, simétrica e aguda, em pacientes internados em unidades de terapia intensiva. Esses pacientes apresentavam dificuldade na retirada da ventilação mecânica além de tetraparesia e reflexos profundos abolidos ${ }^{2}$. O principal fator associado para ocorrência desta polineuropatia é a síndrome de resposta inflamatória sistêmica (SRIS), na maioria das vezes desencadeada por sepse.

No entanto, antecedendo estes relatos, em 1977, MacFarlane e Rosenthal ${ }^{3}$ descreveram pela primeira vez um quadro denominado "miopatia do paciente crítico" em paciente com asma grave que manifestou tetraplegia, após uso de elevadas doses de corticóides e bloqueadores neuromusculares. Posteriormente, surgiram na literatura, inúmeros relatos semelhantes a esse, com diversas nomenclaturas diferentes. Dentre elas miopatia necrotizante da UTI, miopatia tetraplégica aguda, miopatia esferoidal aguda, síndrome pós-paralisia e miopatia de filamentos grossos. Essa síndrome, que provavelmente permanece não adequadamente diagnosticada como "Polineuropatia do Paciente Crítico" (PPC) é usual, mas não obrigatoriamente associada ao uso concomitante de corticosteróide e bloqueadores neuromusculares não-despolarizantes. O diagnóstico diferencial entre as duas condições não é fácil e há grande variabilidade na prevalência, em parte devido à ausência de critérios clínicos rígidos para o diagnóstico ${ }^{4}$.

\section{EPIDEMIOLOGIA}

Garnacho-Montero e col. ${ }^{5}$ realizaram em 2005, um estudo com o objetivo de avaliar o impacto da PPC na duração da ventilação mecânica (VM), depois de controlar a coexistência de fatores de risco para a retirada da ventilação mecânica; como resultado deste estudo, ficou estabelecido que a PPC aumenta o tempo de VM e é preditor independente de insucesso na retirada da ventilação mecânica (pacientes com PPC mantém tempo médio em ventilação mecânica maior do que os que não apresentam com 34 (12-99) dias versus 14 (744) dias e $p<0,001)$. A duração do suporte ventilatório antes da indicação de sua retirada, nos pacientes com PPC, foi maior do que em pacientes que não apresentavam essa complicação. No entanto, o período de retirada da VM também foi maior nos pacientes com PPC; isso se deve a essa anormalidade neurológica e não ao período entre a instituição de VM e o início de sua retirada. Houve aumento da incidência de re-intubação nestes pacientes, além da constatação da PPC como o único fator de risco independente para o insucesso do "desmame" (OR-15,4; 95\% IC, 4,55-52,3; $p<$ $0,001)$. O estudo também mostrou que $41.2 \%$ dos pacientes com PPC necessitaram de re-intubação versus $13,3 \%$ dos que não apresentaram a doença $(p<0,5)$. Em outro estudo, o mesmo grupo de pesquisadores demonstrou, através de uma análise multivariada, a 
presença de fatores de risco importantes associados a PPC, dentre eles a hiperosmolaridade (OR 4,8; IC 95\% $1,05-24,38, p=0,046)$, nutrição parenteral (OR 5,11; IC $95 \%, 1,14-22,88 ; p=0,02)$, uso de bloqueadores neuromusculares (OR 16,32; IC 95\% 1,34-199; $p=0,0008)$, escala de coma de Glasgow abaixo de 10 (OR 24,02; IC 95\% 3,68-156,7; $p=0,001$ ) e por fim a necessidade de diálise (OR 0,02 ; IC 95\% 0,05-0,15, $\mathrm{p}=0,001)^{6}$.

Tepper e col. ${ }^{7}$ avaliaram pacientes que foram admitidos em UTI com choque séptico e analisaram o início da PPC em um grupo de pacientes críticos com alto risco para desenvolvimento da síndrome de disfunção de múltiplos órgãos (DMOS). Os resultados indicaram que a PPC pode ser uma complicação inicial do choque séptico; neste estudo, a PPC apareceu apenas associada à DMOS, levando a crer que esta complicação possa estar integrada à síndrome (OR 7,98; p < 0001). A causa da relação entre DMOS e PPC não foi investigada, no entanto a explicação plausível seria a disfunção entre a oferta e o consumo de oxigênio que afetaria o sistema nervoso periférico ${ }^{7}$.

Segundo alguns autores, pacientes com DMOS têm mortalidade de aproximadamente $50 \%$ e pode chegar à $98 \%$ naqueles que apresentam três ou mais sistemas e ou órgãos em disfunção. As formas mais graves podem estar associadas a uma recuperação relativa, em sua minoria ${ }^{8}$.

De acordo com Jonghe e col. ${ }^{9}$ em estudo realizado em cinco UTI, foi determinado que o sexo feminino e a presença de diabete melito (OR 4,1 $p=0,004$ e OR 3,2 $p=0,05$, respectivamente) estavam significativamente associadas à polineuropatia do paciente crítico, além destes, outros fatores como níveis mais elevados de uréia (OR 1,04; $p=0,1)$, glicose (OR 1,11; $p=0,001$ ) e incidência de infecções (OR 1,9; $p=0,53$ ) também estiveram associados à neuropatia.

\section{FISIOPATOLOGIA E ETIOPATOGENIA}

Sugere-se que a polineuropatia esteja relacionada com a própria fisiopatologia da sepse ${ }^{10-14}$, durante a qual há distúrbios na micro-circulação, perda da auto-regulação dos vasos sanguíneos que suprem os nervos periféricos e a liberação de citocinas, que aumentam a permeabilidade dos vasos, tudo isso resultando em edema endoneural ${ }^{10,12,13}$. Este levaria à hipóxia e conseqüentes déficits resultando em degeneração axonal primária de fibras sensitiva e motora, predominantemente distal em função de seu envolvimento no transporte axonal altamente dependente de energia ${ }^{8,11}$. É possível também que as próprias citocinas, como o fator de necrose tumoral, tenham efeito tóxico direto no nervo periférico.

Essa polineuropatia tem gravidade variável e afeta especialmente os membros inferiores, sendo de maior gravidade na parte distal. Podem ocorrer distúrbios sensitivos leves. Geralmente não há manifestações disautonômicas. O seu início é de difícil reconhecimento, geralmente só sendo possível quando as complicações da sepse ou falência de múltiplos órgãos tenham sido adequadamente controladas ${ }^{11}$.

\section{EVOLUÇÃO E EXAMES COMPLEMENTARES}

A PPC é uma neuropatia axonal aguda desenvolvida durante o tratamento de pacientes graves, apresentando reversão do quadro, assim que a condição crítica em tratamento for controlada. O curso da doença é monofásica e autolimitada, e a recuperação é geralmente boa em pacientes que apresentem a forma leve à moderada da doença ${ }^{8}$.

A eletroneuromiografia é caracterizada como o exame gold standard. Os seus resultados (ENMG) caracterizam-se por potenciais de ações motora e sensitiva de baixa amplitude; velocidade de condução e latências distais relativamente preservadas; pode haver fibrilações e ondas positivas na estimulação com agulha.

O diagnóstico é feito através do exame de eletroneuromiografia, determinando o padrão da polineuropatia axonal, descartando a possibilidade de lesão medular em pacientes que apresentem fraqueza muscular após trauma; a administração de grande quantidade de medicamentos, causando ataque porfírico, para pacientes com porfiria intermitente aguda; pacientes com microabcessos metastáticos que podem causar lesões nervosas periféricas; e a presença de doenças preexistentes descompensadas de músculo, nervo ou motoneurônios em pacientes em que a razão para insuficiência respiratória aguda não esteja aparente. Além disso, também é relevante que a causa de internação do paciente na unidade de terapia intensiva esteja claramente determinada ${ }^{8}$.

O exame do líquido cefalorraquidiano (LCR) é normal ou apresenta leves anormalidades.

A biópsia de nervo mostra degeneração axonal primária sem evidência de inflamação. Segundo Leijten e De Weerd, a disparidade em alguns casos entre os achados patológicos e a gravidade de manifestação clínica é argumento a favor de uma base mais funcional do que anatômica da axonopatia. A recuperação é boa, desde que a doença de base seja controlada ${ }^{15}$. 


\section{TRATAMENTO}

Em relação ao tratamento da polineuropatia do paciente crítico muito ainda se têm a estudar, de tal modo que ainda não se dispõe de nenhum tratamento medicamentoso efetivo. Porém, sob o ponto de vista de seguimento e avaliação multidisciplinar já se pode contar com abordagem fisioterápica efetiva, que auxilie na recuperação destes pacientes.

Um fato importante dentro da organização de um programa fisioterápico, observado por Rich e col., refere-se ao fato do músculo na quadriplegia recorrente da polineuropatia do paciente crítico ser eletricamente inexcitável e permanecer neste estado por dias ou até meses depois da supressão dos medicamentos ${ }^{16}$. Alguns protocolos estão sendo criados para a reabilitação precoce destes pacientes. Protocolo cinesioterápico Laufer em pacientes que fizeram uso prolongado de corticóides e curare - (polineuropatia do paciente crítico) ${ }^{16}$ que consiste das seguintes etapas ou pontos:

1) Teste diário de reflexos;

2) Durante o período de sedação é realizada a Cinesioterapia Motora Passiva segundo respaldo científico do artigo Neural Substrate for the effects of passive training on sensoriomotor cortical representation: a study with functional magnetic resonance imaging in healthy subjects. Eles defendem que o movimento passivo repetido apresenta representação cortical observado no exame de ressonância magnética. Além disto, o trabalho passivo mantém íntegras as estruturas articulares durante o período prolongado de imobilização no leito - realizado na UTI;

3) Quando o paciente inicia a interação com o meio ambiente estabelece-se a Cinesioterapia Motora Ativa Assistida, visando o aumento de força e resistência muscular localizada.

4) Trabalha-se com Facilitação Neuromuscular Proprioceptiva dando ênfase ao reflexo de estiramento e quando necessário utiliza-se a crio-estimulação.

5) Inicia-se a Cinesioterapia Ativa Livre, seguida da Cinesioterapia Resistida, controle de tronco e do ortostatismo.

6) Finaliza-se o trabalho com a deambulação pelo quarto e depois no corredor ${ }^{16}$.

\section{CONCLUSÃO}

A despeito de sua prevalência, na PPC ainda permanecem desconhecidos fatores que claramente associados a sua fisiopatologia bem como adequada terapia para manuseio desta condição. Apesar destas implicações, a maioria dos intensivistas concorda que o desenvolvimento da PPC não deve ser entendido como forma de reduzir a intensidade do tratamento.

\section{REFERÊNCIAS}

01. Bolton CF - The value of diagnostic imaging techniques in the management of diseases of the nervous system. Con Med Assoc J, 1984;130:1425-1426.

02. Druschky A, Herkert M, Radespiel-Troger M et al - Critical illness polyneuropaty: clinical findings and cell culture assay of neurotoxicity assessed by a prospective study. Intensive Care Med, 2001;27:686-693.

03. MacFarlane IA, Rosenthal FD - Severe miopaty after status asthmaticus. Lancet, 1977;2:615.

04. Reis RG, Oliveira ASB - Drogas e sistema nervoso periférico. Rev Neurociências, 1999; VII(3):119-121.

05. Garnacho-Montero J, Amaya-Villar R, Garcia-Garmendia JL et al - Effect of critical illness polyneuropathy on the withdrawal from mechanical ventilation and the length of stay in septic patients. Crit Care Med, 2005;33:349-354.

06. Garnacho-Montero J, Madrazo-Osuna J, Garcia-Garmendia JL et al Critical illness polyneuropathy: risk factors and clinical consequences. A cohort study in septic patients. Intensive Care Med, 2001;27:12881296.

07. Tepper M, Rakic S, Haas JA et al - Incidence and onset of critical illness polyneuropaty in patients with septic shock. Neth J Med, 2000;56:211214.

08. Hund E - Neurological complications of sepsis: critical illness polyneuropathy and myopathy. J Neurol, 2001;248:929-934.

09. Jonghe B, Sharshar T, Lefaucheur JP et al - Paresis acquired in the intensive care unit: a prospective multicenter study. JAMA, 2002;288:28592867.

10. Bolton CF - Sepsis and the systemic inflammatory response syndrome: neuromuscular manifestations. Crit Care Med, 1996;24:1408-1416.

11. Zochodne DW, Bolton CF, Wells GA et al - Critical illness polyneuropathy. A complication of sepsis and multiple organ failure. Brain, 1987;110:819841 .

12. Bolton CF, Young GB, Zochodne DW - The neurological complications of sepsis. Ann Neurol, 1993;33:94-100.

13. Witt NJ. Zochodne DW, Bolton CF et al - Peripheral nerve function in sepsis and multiple organ failure. Chest, 1991;99:176-184

14. Alvim LBAM, Freitas MRG, Nascimento OJM et al - Polineuropatia do paciente crítico: registro de caso. Arq. Neuro-Psiquiatr. [online]. Jun. 1999, vol.57, no. 2A [citado 08 Fevereiro 2006], p.317-322. Disponível em: <http: //www.Scielo.Br/scielo.Php?Script=sci_arttext\&pid=S0004282X1999000200026\&lng=pt\&nrm=iso>. ISSN 0004-282X. Consultado em 16/02/2006.

15. Leijten FS, de Weerd AW. - Critical illness polyneuropathy, facts and controversies. J Peripher Nerv Syst, 1996:1:28-33.

16. Rich MM, Teener JW, Raps EC et al - Muscle is electrically inexcitable in acute quadriplegic myopathy. Neurology, 1996;46:731-736. 